\title{
COMPLEXITY OF TERM REPRESENTATIONS OF FINITARY FUNCTIONS
}

\author{
ERHARD AICHINGER, NEBOJŠA MUDRINSKI, AND JAKUB OPRŠAL
}

\begin{abstract}
The clone of term operations of an algebraic structure consists of all operations that can be expressed by a term in the language of the structure. We consider bounds for the length and the height of the terms expressing these functions, and we show that these bounds are often robust against the change of the basic operations of the structure.
\end{abstract}

\section{Motivation}

Following [BS81], an algebraic structure, or an algebra, is a pair $\mathbf{A}=(A, F)$ where $A$ is a non-empty set called the universe of $\mathbf{A}$, and $F$ is a set of operations on $A$. The operations in $F$ are called the basic operations of $\mathbf{A}$. A language (sometimes called signature, or type) of an algebra $\mathbf{A}$ is a set of symbols, one for each basic operation, together with their arities. An $n$-ary term over the variables $x_{1}, \ldots, x_{n}$ is any properly formed expression using these operation symbols and variables. A natural measure of the complexity of a term is its length as a string, i.e., the total number of both operation and variable symbols it contains. We will denote this quantity by $\operatorname{len}(t)$ and call it the length of the term $t$. Formally, len $(x)=1$ if $x$ is a variable or a nullary operation symbol, and $\operatorname{len}\left(f\left(t_{1}, \ldots, t_{k}\right)\right)=1+\sum_{i=1}^{k} \operatorname{len}\left(t_{i}\right)$ if $f$ is a $k$-ary operation symbol and $t_{1}, \ldots, t_{k}$ are terms. A term $t$ over $x_{1}, \ldots, x_{n}$ naturally induces a function $t^{\mathbf{A}}$ from $A^{n}$ to $A$, which maps a tuple $\left(a_{1}, \ldots, a_{n}\right)$ to the value obtained by substituting $a_{i}$ for each $x_{i}$ and interpreting the operation symbols by the corresponding operations. Next, we measure the complexity of a term function of a finite algebra $\mathbf{A}$ : the length of a term function $t^{\mathbf{A}}$ is the length of the shortest term that induces this function; formally,

$$
\operatorname{len}_{\mathbf{A}}\left(t^{\mathbf{A}}\right)=\min \left\{\operatorname{len}(s): s \text { is a term in the language of } \mathbf{A} \text { with } s^{\mathbf{A}}=t^{\mathbf{A}}\right\} .
$$

From this we define a sequence $\mathbf{l}_{\mathbf{A}}(n)$ whose $n$-th element expresses the worst-case complexity of an $n$-ary term function of $\mathbf{A}$ :

$$
\mathrm{l}_{\mathbf{A}}(n)=\max \left\{\operatorname{len}_{\mathbf{A}}\left(t^{\mathbf{A}}\right): t \text { is an } n \text {-ary term in the language of } \mathbf{A}\right\} .
$$

In other words, $\mathrm{l}_{\mathbf{A}}(n)$ is the smallest number of symbols that is sufficient to write down any $n$-ary term operation of $\mathbf{A}$. In the present paper we study how the asymptotics of the sequence $\mathbf{l}_{\mathbf{A}}(n)$ depends on the properties of the algebra $\mathbf{A}$. The algebras $\mathbf{A}$ and $\mathbf{B}$ are term equivalent if they are defined on the same universe and have the same term operations. Unlike other similar sequences studied in the literature, e.g., the free spectrum, the sequences $l_{\mathbf{A}}$ and $l_{\mathbf{B}}$ may differ even when $\mathbf{A}$ and $\mathbf{B}$ are term equivalent. Nevertheless, in some cases we are able to prove that the asymptotics of these sequences does not change. This motivates the following definition.

Date: October 10, 2018.

Supported by the Austrian Science Fund (FWF):P29931, Research Grant 174018 of the Ministry of Science and Education of the Republic of Serbia, and European Research Council (Grant Agreement no. 681988, CSP-Infinity). 
Definition 1.1. Let $\mathbf{A}$ be a finite algebra of finite type. Then $\mathbf{A}$ is resilient against change of signature, or simply resilient if for every algebra $\mathbf{B}$ of finite type that is term equivalent to $\mathbf{A}$, there exist polynomials $p, q$ such that $\mathrm{l}_{\mathbf{A}}(n) \leq p\left(\mathrm{l}_{\mathbf{B}}(n)\right)$ and $\mathrm{l}_{\mathbf{B}}(n) \leq q\left(\mathrm{l}_{\mathbf{A}}(n)\right)$ for each $n \in \mathbb{N}$.

It is not known to the authors whether there is a finite algebra of finite type which is not resilient against change of signature. We show that two classes of algebras are resilient. In both cases, the sequences $\mathbf{l}_{\mathbf{A}}(n)$ are close to the theoretical lower bound given by a simple counting argument described in the following section. These two cases are primal algebras and supernilpotent algebras in congruence modular varieties. We recall that an algebra $\mathbf{A}$ with a universe $A$ is called primal if every operation on $A$ is a term operation of $\mathbf{A}$.

Theorem 1.2. Let $\mathbf{A}$ be a finite primal algebra of finite type with at least 2 elements. Then there exist positive real numbers $c_{1}, c_{2}$ such that for all $n \in \mathbb{N}, 2^{c_{1} n} \leq \mathrm{l}_{\mathbf{A}}(n) \leq$ $2^{c_{2} n}$.

Corollary 1.3. Every finite primal algebra of finite type is resilient.

The proof of these results is given in Section 3.

The other property, supernilpotency, can be defined by a condition on higher commutators of the algebra. Higher commutators, introduced in [Bul01], generalize binary commutators, whose theory has been described in [FM87]. They have been studied in [AM10] and recently in [Opr16, Moo16, Wir17]. By a result of Kearnes [Kea99], the supernilpotent algebras distinguish themselves among other algebras in a congruence modular variety by having a small number of term operations. For a precise statement of this fact and a self-contained definition of supernilpotency, we refer the reader to Section 4.

Theorem 1.4. Let $\mathbf{A}$ be a finite supernilpotent algebra in a congruence modular variety with at least 2 elements. Then there exists a polynomial $q$ such that for all $n \in \mathbb{N}, n-1 \leq \mathrm{l}_{\mathbf{A}}(n) \leq q(n)$.

Corollary 1.5. Every finite supernilpotent algebra of finite type in a congruence modular variety is resilient.

These results are proved in Section 4. For groups, Horváth and Nehaniv have proved that for each nilpotent group $\mathbf{G}, \mathrm{l}_{\mathbf{G}}(n)$ is bounded from above by a polynomial in $n$, and for simple groups, one has $\mathrm{l}_{\mathbf{G}}(n) \leq C(\mathbf{G}) \cdot n^{8} \cdot|G|^{n}$ where $C(\mathbf{G})$ depends only on the group $\mathbf{G}$ [HN15].

\section{Notation And General Bounds}

We will write $\mathbb{N}$ for the set of positive integers, $\mathbb{N}_{0}=\mathbb{N} \cup\{0\}$, and $\log (n)$ is the logarithm of $n$ in base $e$. For an algebra $\mathbf{A}$, the set of all term operations is denoted by Clo $\mathbf{A}$ and called the clone of term operations of $\mathbf{A}$. Its $n$-ary part is denoted by $\mathrm{Clo}_{n} \mathbf{A} . \mathrm{Clo}_{n} \mathbf{A}$ can be endowed with a structure of an algebra, denoted by $\mathbf{F}_{\mathbf{A}}(n)$, in the same language as $\mathbf{A}$ by setting:

$$
f^{\mathbf{F}_{\mathbf{A}}(n)}\left(t_{1}^{\mathbf{A}}, \ldots, t_{k}^{\mathbf{A}}\right)=\left(f\left(t_{1}, \ldots, t_{k}\right)\right)^{\mathbf{A}},
$$

or equivalently defining the operations coordinatewise, seeing $\mathrm{Clo}_{n} \mathbf{A}$ as a subuniverse of $\mathbf{A}^{A^{n}}$. It is well-known that this algebra is freely generated (in HSP A) by the projections (the set $\left\{x_{1}^{\mathbf{A}}, \ldots, x_{n}^{\mathbf{A}}\right\}$ ). The sequence defined by

$$
\operatorname{Spec}_{\mathbf{A}}(n)=\left|\mathbf{F}_{\mathbf{A}}(n)\right|
$$

for $n \in \mathbb{N}$ is called the free spectrum of $\mathbf{A}$.

We will now introduce another measure for the complexity of a term. Every term can be expressed as a rooted tree whose vertices correspond to the function and variable symbols appearing in it. The variables correspond to leaves, each function 


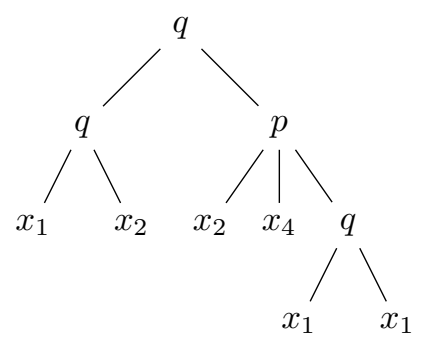

FiguRE 1. Tree representation of term $q\left(q\left(x_{1}, x_{2}\right), p\left(x_{2}, x_{4}, q\left(x_{1}, x_{1}\right)\right)\right)$.

symbol has exactly as many children as is the arity of the symbol, and the children have a (usually implicitly) given order. We call this tree the tree representation of a term $t$. The number of vertices of the tree representing $t$ is exactly len $(t)$. The height of the term $t$, denoted by ht $(t)$, represents the height of the tree representation of $t$, with an adjustment if $t$ contains a nullary operation symbol: Precisely, we define $\mathrm{ht}(t)$ inductively by setting: $\operatorname{ht}(x)=0$ if $x$ is a variable; $h t(f)=1$ if $f$ is a nullary operation symbol; and if $f$ is a $k$-ary operation symbol where $k \in \mathbb{N}$, and $t_{1}, \ldots, t_{k}$ are terms, we set $h t\left(f\left(t_{1}, \ldots, t_{k}\right)\right)=1+\max \left\{h t\left(t_{1}\right), \ldots, h t\left(t_{k}\right)\right\}$. Through the rest of the present paper, we set $\max \emptyset=0$. This will simplify some proofs since it saves a case distinction between nullary and non-nullary operation symbols.

The height of a term function $t^{\mathbf{A}}$ is defined by

$$
\operatorname{ht}_{\mathbf{A}}\left(t^{\mathbf{A}}\right)=\min \left\{\mathrm{ht}(s): s \text { is a term in the language of } \mathbf{A} \text { with } s^{\mathbf{A}}=t^{\mathbf{A}}\right\} .
$$

This allows us to define a second sequence measuring the complexity of terms of an algebra $\mathbf{A}$. For $n \in \mathbb{N}$, let

$$
\mathrm{h}_{\mathbf{A}}(n)=\max \left\{\mathrm{ht}_{\mathbf{A}}\left(t^{\mathbf{A}}\right): t \text { is an } n \text {-ary term in the language of } \mathbf{A}\right\} .
$$

Lemma 2.1. Let $\mathbf{A}$ be a finite algebra of finite type, let $r$ be the number of operation symbols of $\mathbf{A}$ and $m$ be their maximal arity. Then:

(1) $\operatorname{Spec}_{\mathbf{A}}(n)<(n+r+1)^{1_{\mathbf{A}}(n)}$ for all $n \in \mathbb{N}$;

(2) there exists a positive real number $c$ such that for all $n \geq 2$ we have $\operatorname{Spec}_{\mathbf{A}}(n) \leq 2^{c \log (n) 1_{\mathbf{A}}(n)}$

(3) $\mathrm{h}_{\mathbf{A}}(n) \leq \operatorname{Spec}_{\mathbf{A}}(n)$ for all $n \in \mathbb{N}$;

(4) $\mathrm{h}_{\mathbf{A}}(n) \leq \mathrm{l}_{\mathbf{A}}(n)$ for all $n \in \mathbb{N}$;

(5) $\mathrm{l}_{\mathbf{A}}(n) \leq 1+m+\cdots+m^{\mathrm{h}_{\mathbf{A}}(n)} \leq(m+1)^{\mathrm{h}_{\mathbf{A}}(n)}$ for all $n \in \mathbb{N}$.

Proof. (1) By the definition of the sequence $\mathbf{l}_{\mathbf{A}}(n)$, every $n$-ary term operation is given by a term whose representation in prefix notation is a string of length at most $l_{\mathbf{A}}(n)$ using $n$ symbols for variables and $r$ symbols for basic operations. Therefore, there are at most $\sum_{l=1}^{l_{\mathbf{A}}(n)}(n+r)^{l}<(n+r+1)^{l_{\mathbf{A}}(n)}$ term operations of A of arity $n$. Now (2) follows because there is a positive real number $c$ such that $\log _{2}(n+r+1) \leq c \log n$ for all $n \geq 2$.

(3) Let $F \leq \mathbf{A}^{A^{n}}$ be the universe of the free algebra generated by the projections $\pi_{1}, \ldots, \pi_{n}$, i.e., the elements of $F$ are precisely the $n$-ary term functions of $\mathbf{A}$. We consider the following alternative construction of the set $F$ : We start by taking $H_{0}=\left\{\pi_{1}, \ldots, \pi_{n}\right\}$, and then inductively define sets $H_{k}$ by

$$
H_{k+1}=\left\{f^{\mathbf{A}^{A^{n}}}\left(t_{1}, \ldots, t_{m}\right): t_{1}, \ldots, t_{m} \in H_{k}, f \text { is a basic operation of } \mathbf{A}\right\} \cup H_{k}
$$

for $k \in \mathbb{N}_{0}$. If for some $k, H_{k+1}=H_{k}$, then $H_{k}$ is closed under the operations of $\mathbf{A}^{A^{n}}$, therefore a subuniverse, and hence $F=H_{k}$. Let $k_{0}$ be the smallest $k$ such that $H_{k}=H_{k+1}$. Then $F=H_{k_{0}}$. Now, since $\left|H_{k+1}\right| \geq\left|H_{k}\right|+1$ for all $k<k_{0}$, we obtain $|F| \geq k_{0}$. Further, observe that for any $k, H_{k}$ is the set of all $n$-ary term 
operations of $\mathbf{A}$ which can be expressed by a term of depth at most $k$, in other words, $H_{k}=\left\{f^{\mathbf{A}} \in \mathrm{Clo}_{n}(\mathbf{A}): \operatorname{ht}\left(f^{\mathbf{A}}\right) \leq k\right\}$. Therefore, since $H_{k_{0}}=F$, we obtain that $\operatorname{ht}\left(f^{\mathbf{A}}\right) \leq k_{0} \leq|F|$ for any $n$-ary term operation $f^{\mathbf{A}}$ of $\mathbf{A}$, which immediately yields the required inequality.

(4) Let $t$ be an $n$-ary term. Then there is a term $s$ with $\operatorname{len}(s) \leq \mathrm{l}_{\mathbf{A}}(n)$ such that $t^{\mathbf{A}}=s^{\mathbf{A}}$. For every term, we have $\operatorname{len}(s) \geq \operatorname{ht}(s)$, and therefore $\operatorname{ht}(s) \leq \mathrm{l}_{\mathbf{A}}(n)$. This implies $\mathrm{h}_{\mathbf{A}}(n) \leq \mathrm{l}_{\mathbf{A}}(n)$.

(5) We first observe that for every term $t$,

$$
\operatorname{len}(t) \leq 1+m+\cdots+m^{\mathrm{ht}(t)}
$$

because a rooted tree in which every vertex has at most $m$ children has at most $m^{d}$ vertices of depth $d$. In order to obtain an upper bound for $l_{\mathbf{A}}(n)$, we let $t$ be an $n$-ary term. Hence $\operatorname{ht}\left(t^{\mathbf{A}}\right) \leq \mathrm{h}_{\mathbf{A}}(n)$, and hence there is a term $s$ with $\operatorname{ht}(s) \leq \mathrm{h}_{\mathbf{A}}(n)$ and $s^{\mathbf{A}}=t^{\mathbf{A}}$. Now (2.1) implies that len $(s)$, and therefore also len $\mathbf{A}\left(t^{\mathbf{A}}\right)$, is at most $\sum_{i=0}^{\mathrm{h}_{\mathbf{A}}(n)} m^{i}$ which completes the proof of the first inequality. The second inequality is immediate.

For an $n$-ary term $t$ and terms $t_{1}, \ldots, t_{n}$, we write $t\left(t_{1}, \ldots, t_{n}\right)$ for the term obtained by substituting every occurrence of $x_{i}$ in $t$ by $t_{i}$.

Lemma 2.2. Let $n \in \mathbb{N}$, let $\mathcal{L}$ be a language, and let $t, t_{1}, \ldots, t_{n}$ be terms of type $\mathcal{L}$. If $t$ is $n$-ary, then $\operatorname{ht}\left(t\left(t_{1}, \ldots, t_{n}\right)\right) \leq \operatorname{ht}(t)+\max \left\{\operatorname{ht}\left(t_{1}\right), \ldots, \mathrm{ht}\left(t_{n}\right)\right\}$.

Proof. We proceed by induction on $\mathrm{ht}(t)$ : If $t$ is a variable $x_{i}$, then $\operatorname{ht}(t)=0$, and the statement reduces to $\mathrm{ht}\left(t_{i}\right) \leq \max \left\{\mathrm{ht}\left(t_{1}\right), \ldots, \mathrm{ht}\left(t_{n}\right)\right\}$ which is trivial. For the induction step, we assume that $t=f\left(s_{1}, \ldots, s_{k}\right)$ for some operation symbol $f$ and $n$ ary terms $s_{1}, \ldots, s_{k}$. To simplify the formulae below, let $m=\max \left\{\operatorname{ht}\left(t_{1}\right), \ldots, \mathrm{ht}\left(t_{n}\right)\right\}$. Observe that $\mathrm{ht}\left(s_{i}\right) \leq \mathrm{ht}(t)-1$. From the induction hypothesis we obtain

$$
\operatorname{ht}\left(s_{i}\left(t_{1}, \ldots, t_{n}\right)\right) \leq \operatorname{ht}\left(s_{i}\right)+m \leq \mathrm{ht}(t)+m-1
$$

for every $i \in\{1, \ldots, k\}$. Therefore,

$$
\begin{aligned}
\operatorname{ht}\left(t\left(t_{1}, \ldots, t_{n}\right)\right) & =\operatorname{ht}\left(f\left(s_{1}\left(t_{1}, \ldots, t_{n}\right), \ldots, s_{k}\left(t_{1}, \ldots, t_{n}\right)\right)\right) \\
=1 & +\max \left\{\operatorname{ht}\left(s_{1}\left(t_{1}, \ldots, t_{n}\right)\right), \ldots, \operatorname{ht}\left(s_{k}\left(t_{1}, \ldots, t_{n}\right)\right)\right\} \leq \operatorname{ht}(t)+m .
\end{aligned}
$$

Lemma 2.3. Let $\mathbf{A}$ and $\mathbf{B}$ be two term equivalent finite algebras of finite type. Then there exist positive real numbers $c_{1}, c_{2}$ such that for all $n \in \mathbb{N}$,

$$
c_{1} \mathrm{~h}_{\mathbf{B}}(n) \leq \mathrm{h}_{\mathbf{A}}(n) \leq c_{2} \mathrm{~h}_{\mathbf{B}}(n) .
$$

Proof. We first consider the second inequality. We choose $c_{2}$ such that every basic operation $f^{\mathbf{B}}$ of $\mathbf{B}$ can be expressed as a term operation of $\mathbf{A}$ of height at most $c_{2}$; such a $c_{2}$ exists because $\mathbf{B}$ is of finite type and the two algebras are term equivalent. Now, given that $t^{\mathbf{A}}$ is a term operation of $\mathbf{A}$ of arity $n$, and $\mathbf{A}$ and $\mathbf{B}$ are term equivalent, then $t^{\mathbf{A}}$ is also a term operation of $\mathbf{B}$. Therefore, there exists a term $s$ in the language of $\mathbf{B}$ of height at most $\mathrm{h}_{\mathbf{B}}(n)$ such that $s^{\mathbf{B}}=t^{\mathbf{A}}$. We will turn this term into a term $r$ in the language of $\mathbf{A}$ with $r^{\mathbf{A}}=s^{\mathbf{B}}$ by substituting every basic operation symbol by its definition as a term of $\mathbf{A}$ (again, we use that $\mathbf{A}$ and $\mathbf{B}$ are term equivalent). The term $r$ which is obtained this way will be of height at most $c_{2} \mathrm{ht}(s) \leq c_{2} \mathrm{~h}_{\mathbf{B}}(n)$. Formally, we prove the existence of a term $r$ with $r^{\mathbf{A}}=s^{\mathbf{B}}$ and $\operatorname{ht}(r) \leq c_{2} \operatorname{ht}(s)$ by induction on the height of $s$. If $s$ is a variable, we set $r:=s$ and obtain the required inequality from the fact that ht $(r)=\operatorname{ht}(s)=0$. Now assume that $s=f\left(s_{1}, \ldots, s_{k}\right)$ for some $k \in \mathbb{N}_{0}$, some terms $s_{1}, \ldots, s_{k}$ and a basic operation $f$ of B. Then $\operatorname{ht}\left(s_{i}\right) \leq \operatorname{ht}(s)-1$ for all $i$. By the induction hypothesis, we know that there are terms $r_{1}, \ldots, r_{k}$ such that

$$
\operatorname{ht}\left(r_{i}\right) \leq c_{2} \operatorname{ht}\left(s_{i}\right) \leq c_{2}(\operatorname{ht}(s)-1) \text { and } r_{i}^{\mathbf{A}}=s_{i}^{\mathbf{B}}
$$


There is also a term $g$ in the language of $\mathbf{A}$ of height at most $c_{2}$ such that $g^{\mathbf{A}}=f^{\mathbf{B}}$. Let $r=g\left(r_{1}, \ldots, r_{n}\right)$. Then $r^{\mathbf{A}}=g^{\mathbf{A}}\left(r_{1}^{\mathbf{A}}, \ldots, r_{k}^{\mathbf{A}}\right)=f^{\mathbf{B}}\left(s_{1}^{\mathbf{B}}, \ldots, s_{k}^{\mathbf{B}}\right)=s^{\mathbf{B}}$. Moreover, from Lemma 2.2 we obtain that $\operatorname{ht}(r) \leq \operatorname{ht}(g)+\max \left\{\operatorname{ht}\left(r_{i}\right): i \in\{1, \ldots, k\}\right\} \leq$ $c_{2}+c_{2}(\mathrm{ht}(s)-1)=c_{2} \mathrm{ht}(s) \leq c_{2} \mathrm{~h}_{\mathbf{B}}(n)$. This completes the induction step. Therefore $\mathrm{h}_{\mathbf{A}}(n) \leq c_{2} \mathrm{~h}_{\mathbf{B}}(n)$.

For proving the first inequality, we interchange the roles of $\mathbf{A}$ and $\mathbf{B}$ to obtain a $c^{\prime}>0$ such that for all $n \in \mathbb{N}, \mathrm{h}_{\mathbf{B}}(n) \leq c^{\prime} \mathrm{h}_{\mathbf{A}}(n)$. Then the inequality is satisfied with $c_{1}:=1 / c^{\prime}$.

Corollary 2.4. Let $\mathbf{A}$ and $\mathbf{B}$ be two term equivalent finite algebras of finite type. Then there exist positive real numbers $d_{1}, d_{2}$ such that for all $n \in \mathbb{N}$,

$$
d_{1} \log _{2}\left(\mathrm{l}_{\mathbf{B}}(n)\right) \leq \mathrm{l}_{\mathbf{A}}(n) \leq 2^{d_{2} \mathrm{l}_{\mathbf{B}}(n)}
$$

Proof. Let $c_{2}$ be the constant from the previous lemma, i.e., we have $\mathrm{h}_{\mathbf{A}}(n) \leq$ $c_{2} \mathrm{~h}_{\mathbf{B}}(n)$ for all $n \in \mathbb{N}$. Let $m$ be the maximal arity of operation symbols of $\mathbf{A}$, and let $d_{2}:=c_{2} \log _{2}(m+1)$. By Lemma $2.1(5)$, we have $\mathrm{l}_{\mathbf{A}}(n) \leq(m+1)^{\mathrm{h}_{\mathbf{A}}(n)}$. Using the inequality on the height, we obtain

$$
(m+1)^{\mathrm{h}_{\mathbf{A}}(n)} \leq(m+1)^{c_{2} \mathrm{~h}_{\mathbf{B}}(n)}=2^{d_{2} \mathrm{~h}_{\mathbf{B}}(n)} .
$$

Now by Lemma $2.1(4)$, we have $2^{d_{2} \mathrm{~h}_{\mathbf{B}}(n)} \leq 2^{d_{2} \mathrm{l}_{\mathbf{B}}(n)}$, and therefore the second inequality holds. For proving the first inequality, we interchange the roles of $\mathbf{A}$ and $\mathbf{B}$ to obtain a $d^{\prime}>0$ such that for all $n \in \mathbb{N}, \mathrm{l}_{\mathbf{B}}(n) \leq 2^{d^{\prime} \mathrm{h}_{\mathbf{A}}(n)}$. Hence $\log _{2}\left(\mathrm{l}_{\mathbf{B}}(n)\right) \leq$ $d^{\prime} \mathrm{h}_{\mathbf{A}}(n)$. Hence the required inequality is satisfied with $d_{1}:=1 / d^{\prime}$.

We are not aware of finite term equivalent algebras $\mathbf{A}$ and $\mathbf{B}$ of finite type such that there is a positive real number $c$ with $\mathrm{l}_{\mathbf{A}}(n) \geq 2^{c \mathrm{l}_{\mathbf{B}}(n)}$ for all $n \in \mathbb{N}$. A natural example that the change of signatures can make it significantly easier to write down certain functions is given by the commutator operation in a group. Let $\mathbf{A}=\mathbf{A}_{4}$ to be the alternating group on four elements, and let $\mathbf{B}$ be the algebra obtained from A by adding a single binary operation $[\cdot, \cdot]$ expressing the commutator of the two elements, i.e., $[x, y]=x^{-1} y^{-1} x y$. Observe that while the $n$-ary iterated commutator $t_{n}=\left[\ldots\left[\left[x_{1}, x_{2}\right], x_{3}\right], \ldots x_{n}\right]$ has linear length in $n$, the corresponding term of $\mathbf{A}$ (the one obtained by simply substituting the definition of the commutator for each of its appearance) has length more than $2^{n}$. Unfortunately, we are unable to prove that the term functions $t_{n}^{\mathbf{B}}$ cannot be represented by terms in the language of $\mathbf{A}$ whose length would be bounded by a polynomial in $n$. Nevertheless, using [HS12] and under the assumption that $\mathrm{P} \neq \mathrm{NP}$, such terms cannot be produced in polynomial time:

Proposition 2.5. Let $\mathbf{A}:=\left(A_{4}, \cdot,{ }^{-1}, 1\right)$ be the alternating group on four letters, let $\mathbf{B}:=\left(A_{4}, \cdot,^{-1}, 1,[.,].\right)$ be its expansion with the commutator operation $[x, y]:=$ $x^{-1} y^{-1} x y$, let $t_{1}=x_{1}$, and for $n \in \mathbb{N}$, let $t_{n}:=\left[t_{n-1}, x_{n}\right]$. If $\mathrm{P} \neq \mathrm{NP}$, then there is no algorithm which, given $n$, produces a term $s_{n}$ in the language of $\mathbf{A}$ such that $s_{n}^{\mathbf{A}}=t_{n}^{\mathbf{B}}$ and which runs in polynomial time in $n$.

Proof. We derive this result from results of [HS12]: the equation solvability problem for $\mathbf{A}$ is in $\mathrm{P}$ [HS12, Theorem 6]. Furthermore, we will use their reduction of 3colorability to the equation solvability problem for B [HS12, Theorem 13].

Suppose that there is a polynomial time algorithm producing $s_{n}$. We will use this algorithm to reduce 3 -colorability to equation solvability in $\mathbf{A}$. To this end, let $\Gamma=(V, E)$ be a graph with $n$ vertices and $k$ edges, and let $V=\left\{v_{1}, \ldots, v_{n}\right\}$ and $E=\left\{e_{1}, \ldots, e_{k}\right\}$. In [HS12], the authors produce a term $t_{\Gamma}$ in the language of $\mathbf{B}$ of polynomial length in $n$ and $a \in A_{4}$ such that $t_{\Gamma} \approx a$ has a solution for $a \neq 1$ if and only if the graph $\Gamma$ is 3 -colorable. This term is defined by

$$
t_{\Gamma}\left(x_{1}, x_{2}, y_{1}, \ldots, y_{n}\right)=\left[\ldots\left[\left[x_{1}, x_{2}\right], g_{e_{1}}\right], \ldots ., g_{e_{k}}\right],
$$


where $g_{\left(v_{i}, v_{j}\right)}=y_{i} y_{j}^{-1}$. The term $t_{\Gamma}$ is of a polynomial length in $n$ as a term of $\mathbf{B}$. Using this term, Horváth and Szabó reduce 3-colorability to equation solvability in B. We have to do one more step to reduce it to equation solvability in $\mathbf{A}$, that is find a polynomial length term in the language of groups. For that we run the given algorithm to produce a term $s_{k+2}$ in the language of $\mathbf{A}$ such that $s_{k+2}^{\mathbf{A}}=t_{k+2}^{\mathbf{B}}$. Note that this term has to be of polynomial length in $k$ since it is produced by a polynomial time algorithm. Finally, let

$$
s_{\Gamma}\left(x_{1}, x_{2}, y_{1}, \ldots, y_{n}\right)=s_{k+2}\left(x_{1}, x_{2}, g_{e_{1}}, \ldots \ldots, g_{e_{k}}\right) \text {. }
$$

This term can be computed from $s_{k+2}$ in linear time, and since $s_{\Gamma}^{\mathbf{A}}=t_{\Gamma}^{\mathbf{B}}$, the equation $t_{\Gamma} \approx a$ has a solution in $A_{4}$ if and only if $s_{\Gamma} \approx a$ does. Hence $\Gamma$ is 3-colorable if and only if $s_{\Gamma} \approx a$ is solvable. This completes the reduction from 3-colorability to equation solvability in A. By [HS12, Theorem 6], equational solvability in $\mathbf{A}$ is in $\mathrm{P}$. Altogether, we have produced a polynomial time algorithm for 3-colorability. Since 3 -colorability is NP-complete [Kar72], this contradicts the assumption $\mathrm{P} \neq \mathrm{NP}$. Therefore, assuming $\mathrm{P} \neq \mathrm{NP}$, such an algorithm producing $s_{n}$ does not exist.

We will also use the following elementary lemma.

Lemma 2.6. Let $f: \mathbb{N} \rightarrow \mathbb{R}$ be a non-decreasing function, let $n_{0} \in \mathbb{N}, 0<c<1$, and $d>0$ such that $c n_{0} \geq 1$ and for all $n \geq n_{0}$ we have $f(n) \leq f(\lfloor c n\rfloor)+d$. Then for all $n \in \mathbb{N}$, we have $f(n) \leq d \log _{1 / c} n+f\left(n_{0}\right)$.

Proof. We will prove the claim by induction on $n$. For $n \leq n_{0}$, the statement follows from monotonicity of $f$. For the induction step, suppose that $n>n_{0}$ and the statement is true for all $m<n$. We obtain

$$
\begin{aligned}
f(n) \leq f(\lfloor c n\rfloor)+d \leq d \log _{1 / c} & (\lfloor c n\rfloor)+f\left(n_{0}\right)+d \\
& \leq d\left(1+\log _{1 / c}(c n)\right)+f\left(n_{0}\right)=d \log _{1 / c} n+f\left(n_{0}\right)
\end{aligned}
$$

from the induction hypothesis and the monotonicity of the logarithm.

\section{Primal algebras}

In this section, we will consider sums $x_{1}+x_{2}+\cdots+x_{n}$, where + is some binary operation. These sums can be parenthesized in various ways. We will put the parentheses in a way that yield a balanced binary tree.

Definition 3.1. Let $t$ be a binary symbol. We define the sequence $\left(\sigma_{n}^{t}\right)_{n \in \mathbb{N}}$ of terms in the language $\{t\}$ by $\sigma_{1}^{t}\left(x_{1}\right):=x_{1}$ and

$$
\sigma_{n}^{t}\left(x_{1}, \ldots, x_{n}\right):=t\left(\sigma_{\lceil n / 2\rceil}^{t}\left(x_{1}, \ldots, x_{\lceil n / 2\rceil}\right), \sigma_{\lfloor n / 2\rfloor}^{t}\left(x_{\lceil n / 2\rceil+1}, \ldots, x_{n}\right)\right)
$$

for $n \geq 2$.

Lemma 3.2. Let $t$ be a binary symbol in the language of an algebra $\mathbf{A}$ and $0 \in A$ such that $t^{\mathbf{A}}(x, 0)=t^{\mathbf{A}}(0, x)=x$ for all $x \in A$. Then for all $n \in \mathbb{N}$ and $x \in A$, we have $\left(\sigma_{n}^{t}\right)^{\mathbf{A}}(0, \ldots, 0, x, 0, \ldots, 0)=x$.

Proof. By induction on $n$. For $n=1$ the statement is obviously true by the definition of $\left(\sigma_{n}^{t}\right)_{n \in \mathbb{N}}$. Let $n \geq 2$, and let $x \in A$. We first consider the case that $x$ appears at place $i$ with $i>\lceil n / 2\rceil$. Then the equalities $\sigma_{\lfloor n / 2\rfloor}^{t} \mathbf{A}(0, \ldots, 0)=0$

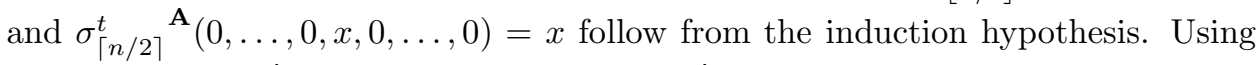
the assumption $t^{\mathbf{A}}(0, x)=x$, we obtain $\left(\sigma_{n}^{t}\right)^{\mathbf{A}}(0, \ldots, 0, x, 0, \ldots, 0)=x$. The case $i \leq\lceil n / 2\rceil$ is done similarly.

The height of a binary balanced tree can be determined from its number of leaves. In our setting, this means:

Lemma 3.3. Let $t$ be a binary symbol. Then $\operatorname{ht}\left(\sigma_{n}^{t}\right)=\left\lceil\log _{2} n\right\rceil$. 
Proof. We prove the statement

$$
\forall k \in \mathbb{N}_{0} \forall n \in \mathbb{N}: 2^{k-1}<n \leq 2^{k} \Rightarrow \operatorname{ht}\left(\sigma_{n}^{t}\right)=k
$$

by induction on $k$. It is clearly true for $k \in\{0,1\}$. Now assume that $k>1$. Since $2^{k-1}<n \leq 2^{k}$, have $2^{k-2}<n / 2 \leq 2^{k-1}$, and therefore, $2^{k-2}<\lceil n / 2\rceil \leq 2^{k-1}$ and $\lfloor n / 2\rfloor \leq 2^{k-1}$. From these inequalities and the induction hypothesis, we obtain that $\operatorname{ht}\left(\sigma_{\lceil n / 2\rceil}^{t}\right)=k-1$ and $\operatorname{ht}\left(\sigma_{\lfloor n / 2\rfloor}^{t}\right) \leq k-1$. Hence, from the definition of $\sigma_{n}^{t}$, we get $\operatorname{ht}\left(\sigma_{n}^{t}\right)=k$. This completes the induction proof. Now we notice that if $2^{k-1}<n \leq 2^{k}$, then $k=\left\lceil\log _{2} n\right\rceil$, which implies the result.

Lemma 3.4. Let $\mathbf{A}$ be a finite algebra that has binary operations + and $\cdot$ as fundamental operations as well as characteristic functions $\chi_{a}$ for all $a \in A$ such that $x+0=0+x=x, x \cdot 1=x$ and $x \cdot 0=0$ for all $x \in A$. If all unary constant operations of $\mathbf{A}$ are term functions then there is a positive real number $d$ such that $\mathrm{h}_{\mathbf{A}}(n) \leq d n$ for all $n \in \mathbb{N}$.

Proof. Let $n \in \mathbb{N}$, let $|A|=m, m \in \mathbb{N}$, let $A=\left\{a_{1}, \ldots, a_{m}\right\}$ and let us denote $\Pi_{i=1}^{n} \chi_{\alpha_{i}}\left(x_{i}\right)=\left(\ldots\left(\chi_{\alpha_{1}}\left(x_{1}\right) \cdot \chi_{\alpha_{2}}\left(x_{2}\right)\right) \cdot \ldots\right) \cdot \chi_{\alpha_{n}}\left(x_{n}\right)$ by $\chi_{\alpha_{1}}\left(x_{1}\right) \cdots \chi_{\alpha_{n}}\left(x_{n}\right)$ for all $\alpha_{1}, \ldots, \alpha_{n} \in A$ and $x_{1}, \ldots, x_{n} \in A$. By Lemma 3.2 we have that arbitrary term function $f$ can be represented as

$$
\sigma_{m^{n}}^{+}\left(f\left(a_{1}, \ldots, a_{1}\right) \cdot \chi_{a_{1}}\left(x_{1}\right) \cdots \chi_{a_{1}}\left(x_{n}\right), \ldots, f\left(a_{m}, \ldots, a_{m}\right) \cdot \chi_{a_{m}}\left(x_{1}\right) \cdots \chi_{a_{m}}\left(x_{n}\right)\right) .
$$

We note that $\operatorname{ht}(x+y)=\operatorname{ht}(x \cdot y)=1$ and $\operatorname{ht}\left(\chi_{a_{i}}(x)\right)=1$ for all $i \in\{1, \ldots, m\}$. Let $s$ be the maximal height of the terms that represent constant functions. Now we can calculate the height of (3.1). Using Lemma 3.3, we have ht $\left(\sigma_{m^{n}}^{+}\right) \leq\left\lceil\log _{2}|A|^{n}\right\rceil$. Using the definition of the height we obtain

$$
\operatorname{ht}\left(f\left(\alpha_{1}, \ldots, \alpha_{n}\right) \cdot \Pi_{i=1}^{n} \chi_{\alpha_{i}}\left(x_{i}\right)\right)=1+\max \left\{\operatorname{ht}\left(f\left(\alpha_{1}, \ldots, \alpha_{n}\right)\right), \operatorname{ht}\left(\Pi_{i=1}^{n} \chi_{\alpha_{i}}\left(x_{i}\right)\right)\right\}
$$

and $\operatorname{ht}\left(\Pi_{i=1}^{n} \chi_{\alpha_{i}}\left(x_{i}\right)\right)=n$ for all $\alpha_{1}, \ldots, \alpha_{n} \in A$. Hence, for all $n \in \mathbb{N}$, we have

$$
\operatorname{ht}\left(f\left(\alpha_{1}, \ldots, \alpha_{n}\right) \cdot \Pi_{i=1}^{n} \chi_{\alpha_{i}}\left(x_{i}\right)\right) \leq 1+\max \{s, n\} .
$$

Hence there is $c \in \mathbb{N}$ such that $\operatorname{ht}\left(f\left(\alpha_{1}, \ldots, \alpha_{n}\right) \cdot \Pi_{i=1}^{n} \chi_{\alpha_{i}}\left(x_{i}\right)\right) \leq c n$ for all $n \in \mathbb{N}$. Therefore, for each $n \in \mathbb{N}$, by Lemma 2.2 the height of (3.1) is at most $\left\lceil\log _{2}|A|^{n}\right\rceil+$ $c n$. There is a positive real $d$ such that for all $n \in \mathbb{N},\left\lceil\log _{2}|A|^{n}\right\rceil+c n \leq d n$. Hence $\mathrm{h}_{\mathbf{A}}(n) \leq d n$.

Proposition 3.5. Let $\mathbf{A}=(A, F)$ be a finite primal algebra of finite type, and let $n \in \mathbb{N}$. Then there is a positive real number $c$ such that $\mathrm{l}_{\mathbf{A}}(n) \leq 2^{\text {cn }}$ for all $n \in \mathbb{N}$.

Proof. Let $\mathbf{A}$ be a primal algebra and let $u$ be the maximal arity of its fundamental operations. By [KP01, Theorem 3.1.5] every primal algebra $\mathbf{A}=(A, F)$ such that $A=\left\{a_{1}, \ldots, a_{m}\right\}$, is term equivalent to $\mathbf{B}=\left(A,+, \cdot, \chi_{a_{1}}, \ldots, \chi_{a_{m}}, 0,1\right)$ where $\chi_{a_{1}}, \ldots, \chi_{a_{m}}$ are characteristic functions, 0 and 1 are elements from $A$ and + and - are binary operations such that $x+0=0+x=x, x \cdot 1=x, x \cdot 0=0$ for all $x \in A$ and every constant function is a term function of $\mathbf{B}$. By Lemma 2.3, there is a positive real number $a$ such that $\mathrm{h}_{\mathbf{A}}(n) \leq a \mathrm{~h}_{\mathbf{B}}(n)$ for all $n \in \mathbb{N}$. Using Lemma 3.4 , we obtain a positive real $d$ such that $\mathrm{h}_{\mathbf{A}}(n) \leq d n$ for all $n \in \mathbb{N}$. Using Lemma 2.1 we have $l_{\mathbf{A}}(n) \leq(u+1)^{d n}$, and thus we can find a positive real $c$ such that $\mathrm{l}_{\mathbf{A}}(n) \leq 2^{\text {cn }}$ for all $n \in \mathbb{N}$.

Proof of Theorem 1.2. For every primal algebra $\mathbf{A}$ and $n \in \mathbb{N}$ we have $\operatorname{Spec}_{\mathbf{A}}(n)=$ $|A|^{|A|^{n}}$. Now the first inequality follows from Lemma 2.1 (2). The second inequality is given in Proposition 3.5. 


\section{Supernilpotent ALGebras}

The notion of supernilpotency was introduced in [AE06, Definition 4.1] for expansions of groups, and in its general form in [AM10, Definition 7.1]. It is closely related to Bulatov's higher commutators introduced in [Bul01].

Definition 4.1. An algebra $\mathbf{A}$ is said to be supernilpotent of degree $n$ if it satisfies the commutator identity $\left[1_{\mathbf{A}}, \ldots, 1_{\mathbf{A}}\right]=0_{\mathbf{A}}$ where the commutator has arity $n+1$.

The commutator identity in the above definition is described by the following term condition: for all $i=1, \ldots, n+1, k_{i} \in \mathbb{N}, \mathbf{a}_{i}, \mathbf{b}_{i} \in A^{k_{i}}$ with $\mathbf{a}_{i} \neq \mathbf{b}_{i}$, and all terms $t$ of arity $\sum k_{i}$ that satisfy

$$
t\left(\mathbf{x}_{1}, \ldots, \mathbf{x}_{n}, \mathbf{a}_{n+1}\right)=t\left(\mathbf{x}_{1}, \ldots, \mathbf{x}_{n}, \mathbf{b}_{n+1}\right)
$$

for all choices of $\mathbf{x}_{i}$ 's between $\mathbf{a}_{i}$ and $\mathbf{b}_{i}$ except the case where all are $\mathbf{b}_{i}$, we have

$$
t\left(\mathbf{b}_{1}, \ldots, \mathbf{b}_{n}, \mathbf{a}_{n+1}\right)=t\left(\mathbf{b}_{1}, \ldots, \mathbf{b}_{n}, \mathbf{b}_{n+1}\right) .
$$

Recently, Moorhead [Moo16] provided a condition using two terms that is equivalent to the 'one term' condition given by Bulatov in the case that the algebra lies in a congruence modular variety. This gives another simple description of supernilpotency: loosely speaking, for a fixed $\mathbf{a}_{i}$ and $\mathbf{b}_{i}$, the value of any term $s$ on the tuple $\left(\mathbf{b}_{1}, \ldots, \mathbf{b}_{n+1}\right)$ is determined by its values on all other tuples consisting of $\mathbf{a}_{i}$ and $\mathbf{b}_{i}$ in the right order. The next theorem, which is based on the results in [Opr16], shows that this unique value can be obtained as a result of a certain $\left(2^{n}-1\right)$-ary term (a strong cube term [Opr16, p. 375]) applied to the values of all the other tuples. We recall that every algebra $\mathbf{A}$ with a Mal'cev term $q$ has a strong $n$-cube term $q_{n}$ for every $n>1$, and moreover such a term can be obtained recursively from the Mal'cev term by $q_{2}(x, y, z)=q(y, x, z)$ and

$$
q_{n+1}\left(x_{0}, \ldots, x_{2^{n+1}-1}\right)=q_{2}\left(q_{n}\left(x_{0}, \ldots, x_{2^{n}-2}\right), x_{2^{n}-1}, q_{n}\left(x_{2^{n}}, \ldots, x_{2^{n+1}-2}\right)\right) .
$$

Also, by [Opr16, Lemma 4.1], for every $n \geq 2$, every algebra with a strong $n$-cube term has a Mal'cev term. By a polynomial term of the algebra A, we understand a term of the algebra $\mathbf{A}^{*}$, which is the expansion of $\mathbf{A}$ with one nullary constant operation for every element of $A$.

Theorem 4.2. Let $n \geq 2$ and let $\mathbf{A}$ be an algebra with a strong $n$-cube term $q_{n}$. Then the following are equivalent

(1) A is supernilpotent of degree $n-1$;

(2) for all $m_{1}, \ldots, m_{n} \in \mathbb{N}$, for all terms $t$ of arity $m=m_{1}+\cdots+m_{n}$, and for all $\mathbf{a}_{1}, \mathbf{b}_{1} \in A^{m_{1}}, \ldots, \mathbf{a}_{n}, \mathbf{b}_{n} \in A^{m_{n}}$, we have

$q_{n}^{\mathbf{A}}\left(t^{\mathbf{A}}\left(\mathbf{a}_{1}, \ldots, \mathbf{a}_{n}\right), t^{\mathbf{A}}\left(\mathbf{b}_{1}, \mathbf{a}_{2}, \ldots, \mathbf{a}_{n}\right), \ldots, t^{\mathbf{A}}\left(\mathbf{a}_{1}, \mathbf{b}_{2}, \ldots, \mathbf{b}_{n}\right)\right)=t^{\mathbf{A}}\left(\mathbf{b}_{1}, \mathbf{b}_{2}, \ldots, \mathbf{b}_{n}\right) ;$

(3) for all $n$-ary polynomial terms $t$ and all $a_{1}, b_{1}, \ldots, a_{n}, b_{n} \in A$, we have

$$
q_{n}^{\mathbf{A}}\left(t^{\mathbf{A}}\left(a_{1}, \ldots, a_{n}\right), t^{\mathbf{A}}\left(b_{1}, a_{2}, \ldots, a_{n}\right), \ldots, t^{\mathbf{A}}\left(a_{1}, b_{2}, \ldots, b_{n}\right)\right)=t^{\mathbf{A}}\left(b_{1}, b_{2}, \ldots, b_{n}\right) .
$$

The proof heavily relies on the properties of the relation $\Delta\left(\alpha_{1}, \ldots, \alpha_{n}\right)$. In the case $\mathbf{A}$ is a Mal'cev algebra, this relation of arity $2^{n}$ is described in [Opr16, Lemma 3.3] by

$$
\begin{aligned}
& \Delta\left(\alpha_{1}, \ldots, \alpha_{n}\right)=\left\{\left(t\left(\mathbf{a}_{1}, \ldots, \mathbf{a}_{n}\right), t\left(\mathbf{b}_{1}, \mathbf{a}_{2}, \ldots, \mathbf{a}_{n}\right), \ldots, t\left(\mathbf{b}_{1}, \mathbf{b}_{2}, \ldots, \mathbf{b}_{n}\right)\right) \mid\right. \\
& \text { for all } i \in\{1,2, \ldots, n\}: m_{i} \in \mathbb{N}_{0}, \mathbf{a}_{i}, \mathbf{b}_{i} \in A^{m_{i}},\left(\mathbf{a}_{i}, \mathbf{b}_{i}\right) \in \alpha_{i}^{m_{i}}, \\
& \text { and } \left.t \in \mathrm{Clo}_{\sum_{i=1}^{n} m_{i}} \mathbf{A}\right\} .
\end{aligned}
$$

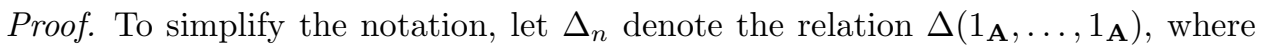
$1_{\mathbf{A}}$ appears $n$ times, and let $[1]_{n}$ denote the $n$-ary commutator $\left[1_{\mathbf{A}}, \ldots, 1_{\mathbf{A}}\right]$.

$(1) \Rightarrow(2)$ : This implication is a consequence of [Opr16, Lemma 4.2]. First, observe that $\left(t^{\mathbf{A}}\left(\mathbf{a}_{1}, \ldots, \mathbf{a}_{n}\right), t^{\mathbf{A}}\left(\mathbf{b}_{1}, \mathbf{a}_{2} \ldots, \mathbf{a}_{n}\right), \ldots, t^{\mathbf{A}}\left(\mathbf{b}_{1}, \mathbf{b}_{2} \ldots, \mathbf{b}_{n}\right)\right) \in \Delta_{n}$. From 
the mentioned lemma, we get that the last element of this tuple is $[1]_{n}$-related to the result of $q_{n}$ applied to all the previous elements. But since $\mathbf{A}$ is supernilpotent of degree $n$, and therefore $[1]_{n}=0_{\mathbf{A}}$, this gives the desired identity.

$(2) \Rightarrow(3)$ : For the $n$-ary polynomial term $t\left(x_{1}, \ldots, x_{n}\right)$, there is a term $s$ in the language of $\mathbf{A}$ and there are $c_{1}, \ldots, c_{m} \in A$ such that

$$
t\left(x_{1}, \ldots, x_{n}\right)=s\left(x_{1}, \ldots, x_{n}, c_{1}, \ldots, c_{m}\right) .
$$

Now we apply (2) for the term $s$ and for $\mathbf{a}_{1}:=a_{1}, \mathbf{b}_{1}:=b_{1}, \ldots, \mathbf{a}_{n-1}:=a_{n-1}$, $\mathbf{b}_{n-1}:=b_{n-1}, \mathbf{a}_{n}:=\left(a_{n}, c_{1}, \ldots, c_{m}\right), \mathbf{b}_{n}:=\left(b_{n}, c_{1}, \ldots, c_{m}\right)$.

$(3) \Rightarrow(1)$ : We will prove that the condition (3) implies that for any $n$-tuple of principal congruences $\theta_{1}, \ldots, \theta_{n}$, we have $\left[\theta_{1}, \ldots, \theta_{n}\right]=0_{\mathbf{A}}$. The claim then follows from join distributivity of the higher commutator. Suppose that $\theta_{i}=\operatorname{Cg}\left(a_{i}, b_{i}\right)$ for all $i$. We know that the relation $\Delta\left(\theta_{1}, \ldots, \theta_{n}\right)$ [Opr16, Lemma 3.3] consists of tuples of the form

$$
\left(t\left(\mathbf{a}_{1}, \ldots, \mathbf{a}_{n}\right), t\left(\mathbf{b}_{1}, \mathbf{a}_{2}, \ldots, \mathbf{a}_{n}\right), \ldots, t\left(\mathbf{b}_{1}, \mathbf{b}_{2}, \ldots, \mathbf{b}_{n}\right)\right)
$$

where $\mathbf{a}_{i} \equiv_{\theta_{i}} \mathbf{b}_{i}$ and $t$ is a term operation of $\mathbf{A}$. Since $\mathbf{A}$ is a Mal'cev algebra, and therefore any reflexive binary compatible relation is a congruence, and since $\delta_{i}$ is generated by $\left(a_{i}, b_{i}\right)$, every pair $(c, d) \in \theta_{i}$ is of the form $\left(p^{\mathbf{A}}\left(a_{i}\right), p^{\mathbf{A}}\left(b_{i}\right)\right)$, where $p$ is a polynomial term of $\mathbf{A}$. Hence, $\mathbf{a}_{i}$ and $\mathbf{b}_{i}$ are of the form $\left(t_{i 1}\left(a_{i}\right), \ldots, t_{i m_{i}}\left(a_{i}\right)\right)$ and $\left(t_{i 1}\left(b_{i}\right), \ldots, t_{i m_{i}}\left(b_{i}\right)\right)$ for some unary polynomial operations $t_{i j}$. By composing these polynomials with $t$, we obtain

$$
\begin{array}{r}
\Delta\left(\theta_{1}, \ldots, \theta_{n}\right)=\left\{\left(p\left(a_{1}, \ldots, a_{n}\right), p\left(b_{1}, a_{2}, \ldots, a_{n}\right), \ldots, p\left(b_{1}, b_{2}, \ldots, b_{n}\right)\right) \mid\right. \\
p \text { is an an } n \text {-ary polynomial operation of } \mathbf{A}\} .
\end{array}
$$

By combining this observation with (3), we obtain that the last coordinate of a tuple in $\Delta\left(\theta_{1}, \ldots, \theta_{n}\right)$ is determined by the other coordinates, therefore by [Opr16, Theorem 1.2], we get that $\left[\theta_{1}, \ldots, \theta_{n}\right]=0_{\mathbf{A}}$, as required.

It is a consequence of [BM14, Lemma 2.7] (which builds upon Lemma 14.6 of [FM87]) that a finite supernilpotent algebra in a congruence permutable variety has a finitely generated clone of term operations. Theorem 4.2 provides another way of establishing this fact.

Corollary 4.3 (cf. [BM14, Lemma 2.7]). Let $n \in \mathbb{N}$, and let A be a supernilpotent Mal'cev algebra of degree $n$. Then the clone of term operations is generated by the Mal'cev term operation together with all term operations of arity at most $n+1$.

Proof. By induction on $k$, we show that every $k$-ary term operation of $\mathbf{A}$ can be generated. We use [Opr16, Lemma 4.1] to produce a strong cube term $q_{n+1}$ of arity $2^{n+1}-1$ for $\mathbf{A}$. Let $k \geq n+2$, and let $f\left(x_{1}, x_{2}, \ldots, x_{k}\right)$ be a $k$-ary term operation. We set $\mathbf{b}_{1}=x_{1}, \ldots, \mathbf{b}_{n}=x_{n}, \mathbf{b}_{n+1}=\left(x_{n+1}, x_{n+2}, \ldots, x_{k}\right), \mathbf{a}_{1}=\cdots=\mathbf{a}_{n}=x_{n+1}$, and $\mathbf{a}_{n+1}$ to the $(k-n)$-tuple $\left(x_{n+1}, \ldots, x_{n+1}\right)$. Theorem $4.2(2)$ implies

$$
\begin{array}{r}
q_{n+1}^{\mathbf{A}}\left(f\left(x_{n+1}, x_{n+1}, \ldots, x_{n+1}, \ldots, x_{n+1}\right), f\left(x_{1}, x_{n+1}, \ldots, x_{n+1}, \ldots, x_{n+1}\right), \ldots\right. \\
\left.f\left(x_{n+1}, x_{2}, \ldots, x_{n+1}, \ldots, x_{k}\right)\right)=f\left(x_{1}, \ldots, x_{k}\right) .
\end{array}
$$

Each of the $2^{n+1}-1$ arguments of $q_{n+1}^{\mathbf{A}}$ contains at least two occurrences of $x_{n+1}$ and is therefore of essential arity at most $k-1$. By the induction hypothesis, each of these arguments describes a function that lies in the clone generated by the Mal'cev operation and the $(n+1)$-ary functions. Since $q_{n+1}$ is composed from the Mal'cev term, $f$ can be generated by the Mal'cev term and functions of arity at most $n+1$.

This generalizes [AM10, Proposition 6.18] to clones that do not contain all constant operations. In contrast to the constantive case, term functions of arity $n$ 
may not suffice: as an example consider the clone $C$ on the set $M_{2 \times 2}\left(\mathbb{Z}_{2}\right)$ of $2 \times 2$ matrices over $\mathbb{Z}_{2}$ that contains all functions $\left(X_{1}, \ldots, X_{k}\right) \mapsto \sum_{i=1}^{k} A_{i} X_{i}$ with $A_{1}, \ldots, A_{k} \in M_{2 \times 2}\left(\mathbb{Z}_{2}\right)$ and $\sum_{i=1}^{k} A_{i}=1$. Then the algebra $\mathbf{A}=\left(M_{2 \times 2}\left(\mathbb{Z}_{2}\right), C\right)$ is 1-supernilpotent, but $C$ is not generated by the identity mapping and the unique Mal'cev operation in the clone.

The condition (2) in Theorem 4.2 also provides an explicit, though infinite, set of identities that defines supernilpotency in a Mal'cev variety:

Corollary 4.4. Let $\mathcal{V}$ be a variety with a strong cube term $q_{n}$. Then the class of all supernilpotent algebras of degree $n$ forms a subvariety of $\mathcal{V}$. This subvariety is defined by the collection of identities of the form

$$
q_{n}\left(t\left(\mathbf{x}_{1}, \ldots, \mathbf{x}_{n}\right), t\left(\mathbf{y}_{1}, \mathbf{x}_{2}, \ldots, \mathbf{x}_{n}\right), \ldots, t\left(\mathbf{x}_{1}, \mathbf{y}_{2}, \ldots, \mathbf{y}_{n}\right)\right) \approx t\left(\mathbf{y}_{1}, \mathbf{y}_{2}, \ldots, \mathbf{y}_{n}\right),
$$

where $t$ is a term of arity $k \geq n$ and $k_{1}, \ldots, k_{n} \in \mathbb{N}$ are such that $k_{1}+\cdots+k_{n}=k$. Here, $\mathbf{x}_{i}$ and $\mathbf{y}_{i}$ denote the tuples of variables $\left(x_{i 1}, \ldots, x_{i k_{i}}\right)$ and $\left(y_{i 1}, \ldots, y_{i k_{i}}\right)$, respectively.

We will now use these identities to express terms of higher arity using the strong cube term $q_{k}$ and terms of smaller arity. This method allows us to prove that there is a logarithmic bound on the sequence $\mathrm{h}_{\mathbf{A}}(n)$ for every supernilpotent finite algebra with a Mal'cev term, and as a consequence, we obtain a polynomial bound on $\mathbf{l}_{\mathbf{A}}(n)$.

Theorem 4.5. Let $\mathbf{A}$ be a finite supernilpotent Mal'cev algebra. Then there exist positive real numbers $c_{1}, c_{2}$ such that for all $n \in \mathbb{N}, \mathrm{h}_{\mathbf{A}}(n) \leq c_{1} \log n+c_{2}$.

Proof. Let $k$ be 1 plus the degree of supernilpotency of $\mathbf{A}$, and let $q_{k}$ be a strong cube term of $\mathbf{A}$. Further assume that $\mathrm{ht}\left(q_{k}\right)=d$. We will prove that there is a constant $c<1$ such that for any large enough $n$, we have $\mathrm{h}_{\mathbf{A}}(n) \leq d+\mathrm{h}_{\mathbf{A}}(\lfloor c n\rfloor)$. To do that, we start with a term $f$ in the language of $\mathbf{A}$ of high-enough arity $n$, and we will group its variables into $k$ pieces of almost the same length, and then use the identity from item (2) in Theorem 4.2 to replace $f$ by a composition of the strong cube term $q_{k}$ with terms of arity lower than $n$. More precisely, let $n=q k+r$ where $r<k, q>1$. We group the variables of $f$ into $r$ many $(q+1)$-tuples $\mathbf{x}_{1}, \ldots, \mathbf{x}_{r}$ and $(k-r)$ many $q$-tuples $\mathbf{x}_{r+1}, \ldots, \mathbf{x}_{k}$ so that $\mathbf{x}_{1}=\left(x_{1}, \ldots, x_{i_{1}}\right), \mathbf{x}_{2}=\left(x_{i_{1}+1}, \ldots, x_{i_{2}}\right)$, etc. We take a new variable $y$, and for $i \in\{1, \ldots, k\}$, we let $\mathbf{y}_{i}$ denote the tuple $(y, \ldots, y)$ of the same length as $\mathbf{x}_{i}$ (i.e., $\mathbf{y}_{i}$ is a $(q+1)$-tuple for $i \leq r$ and a $q$-tuple for $i>r$ ). Now applying the condition (2) of Theorem 4.2, we get that

$$
f\left(\mathbf{x}_{1}, \ldots, \mathbf{x}_{k}\right) \approx q_{k}\left(f\left(\mathbf{y}_{1}, \mathbf{x}_{2}, \ldots, \mathbf{x}_{k}\right), \ldots, f\left(\mathbf{x}_{1}, \mathbf{y}_{2}, \ldots, \mathbf{y}_{k}\right)\right)
$$

is satisfied in $\mathbf{A}$. The right hand side is an application of $q_{k}$ on terms obtained from $f$ by substituting one or more of $\mathbf{x}_{i}$ 's by $\mathbf{y}_{i}$. The maximal arity of these $2^{k}-1$ terms is obtained, e.g., when only $\mathbf{x}_{k}$ is substituted by $\mathbf{y}_{k}$. In this case, omitting $\mathbf{x}_{k}$ reduces the arity of $f$ by $q=\left\lfloor\frac{n}{k}\right\rfloor$ and adds 1 for the new variable $y$. Hence, each of the $2^{k}-1$ arguments of $q_{k}$ in (4.1) contains at most $n+1-\left\lfloor\frac{n}{k}\right\rfloor$ many different variables. For each of these $2^{k}-1$ arguments, we pick a term $u_{i}$ of height at most $\mathrm{h}_{\mathbf{A}}\left(n+1-\left\lfloor\frac{n}{k}\right\rfloor\right)$ representing the same function on A. From Lemma 2.2, we obtain that $q_{k}\left(u_{1}, \ldots, u_{2^{k}-1}\right)$ is a term of height at most $d+\mathrm{h}_{\mathbf{A}}\left(n+1-\left\lfloor\frac{n}{k}\right\rfloor\right)$ that induces the same function on $\mathbf{A}$ as $f$. Therefore,

$$
\mathrm{h}_{\mathbf{A}}(n) \leq d+\mathrm{h}_{\mathbf{A}}\left(n+1-\left\lfloor\frac{n}{k}\right\rfloor\right)
$$

for every $n \in \mathbb{N}$. We choose $\epsilon \in \mathbb{R}$ such that $0<\epsilon<1 / k$, we set $c=1-1 / k+\epsilon$, and let $n_{0}>k$ be big enough so that $\epsilon n_{0} \geq 2$ and $c n_{0} \geq 1$. Then for any $n \geq n_{0}$, we have

$$
n+1-\left\lfloor\frac{n}{k}\right\rfloor<n-\frac{n}{k}+2 \leq\left(1-\frac{1}{k}+\epsilon\right) n=c n .
$$


Therefore, $\mathrm{h}_{\mathbf{A}}(n) \leq d+\mathrm{h}_{\mathbf{A}}(\lfloor c n\rfloor)$ for any $n \geq n_{0}$. From Lemma 2.6, we obtain that $\mathrm{h}_{\mathbf{A}}(n) \leq d \log _{1 / c} n+\mathrm{h}_{\mathbf{A}}\left(n_{0}\right)$ for all $n \in \mathbb{N}$. Choosing $c_{1}:=d / \log (1 / c)$ and $c_{2}=\mathrm{h}_{\mathbf{A}}\left(n_{0}\right)$ we obtain the required result.

Corollary 4.6. Let $\mathbf{A}$ be a finite supernilpotent Mal'cev algebra, then there exist an integer $k>0$ and a positive real $c$ such that for all $n \in \mathbb{N}, \mathrm{l}_{\mathbf{A}}(n) \leq c n^{k}$.

Proof. The algebra A need not be of finite type. However, by Corollary 4.3, its clone of term operations is finitely generated. Therefore, we can choose a finite subset of the fundamental operations of $\mathbf{A}$ that generates all other fundamental operations, and we let $\mathbf{A}^{\prime}$ be the reduct of $\mathbf{A}$ with only these finitely many fundamental operations; let $m$ be their maximal arity. By Theorem 4.5, we have $\mathrm{h}_{\mathbf{A}^{\prime}}(n) \leq c_{1} \log (n)+c_{2}$. Now Lemma 2.1 yields $\mathrm{l}_{\mathbf{A}^{\prime}}(n) \leq(m+1)^{\mathrm{h}_{\mathbf{A}^{\prime}}(n)}$, and thus there is a positive real $c$ and $k \in \mathbb{N}$ such that for all $n \in \mathbb{N}, l_{\mathbf{A}^{\prime}}(n) \leq c n^{k}$. We clearly have $\mathrm{l}_{\mathbf{A}}(n) \leq \mathrm{l}_{\mathbf{A}^{\prime}}(n)$ for all $n \in \mathbb{N}$ which implies the result.

Proof of Theorem 1.4. Since the variety generated by $\mathbf{A}$ is locally finite and has a weak difference term, using [Wir17, Theorem 4.8] and its proof, we get that $\mathbf{A}$ has a Mal'cev term. Corollary 4.6 now yields the second inequality. For the first equality, we show that for every $k \in \mathbb{N}_{0}$, there is an $(2 k+1)$-ary term $t_{k}$ such that $t^{\mathbf{A}}$ depends on all of its arguments. To this end, let $m$ be a Mal'cev term, let $t_{0}\left(x_{1}\right)=x_{1}$, and $t_{k}\left(x_{1}, \ldots, x_{2 k+1}\right)=m\left(t_{k-1}\left(x_{1}, \ldots, x_{2 k-1}\right), x_{2 k}, x_{2 k+1}\right)$ for $k \in \mathbb{N}$. Let $a, b$ be different elements of $A$. We consider $y:=t_{k}^{\mathbf{A}}(a, \ldots, a, b, \ldots, b)$ where the first $2 k+1-l$ arguments are $a$ and the remaining $l$ arguments are set to $b$. Then $y=a$ if $l$ is even, and $y=b$ otherwise. This proves that $t_{k}$ depends on all of its $2 k+1$ arguments. We will now show $\mathbf{l}_{\mathbf{A}}(n) \geq n-1$ : if $n$ is even, then $t_{(n-2) / 2}^{\mathbf{A}}\left(x_{1}, \ldots, x_{n-1}\right)$ depends on $n-1$ arguments. Hence every term representing $t_{(n-2) / 2}^{\mathbf{A}}$ must contain at least $n-1$ variables, and is thus of length at least $n-1$. If $n$ is odd, then $t_{(n-1) / 2}^{\mathbf{A}}\left(x_{1}, \ldots, x_{n}\right)$ depends on all of its $n$ arguments, and thus $\operatorname{len}_{\mathbf{A}}\left(t_{(n-1) / 2}^{\mathbf{A}}\right) \geq n$.

In the rest of this section, we give an argument that out of finite algebras in congruence modular varieties, only supernilpotent ones have a polynomial bound on the length of term functions. This argument is based on a description of the sequence $\operatorname{Spec}_{\mathbf{A}}(n)$. A rough asymptotic behavior of this sequence for congruence modular algebras have been first described by Kearnes in [Kea99]. He proved that an algebra of finite type in a congruence modular variety has a doubly exponential lower bound if and only if it is not a product of prime-power order nilpotent algebras which is now known to be equivalent to being supernilpotent [AM10]. We present a refinement of this result which is given by a combination of several different sources.

Proposition 4.7. Let $\mathbf{A}$ be a finite algebra in a congruence modular variety, and let $k \in \mathbb{N}$. Then $\mathbf{A}$ is $k$-supernilpotent if and only if there is a polynomial $p$ of degree $k$ such that for all $n \in \mathbb{N}$, $\operatorname{Spec}_{\mathbf{A}}(n) \leq 2^{p(n)}$.

Proof. For the "if"-part, first observe that Theorem 9.18 of [HM88] implies that the variety $\mathcal{V}(\mathbf{A})$ omits types $\mathbf{1}$ and $\mathbf{5}$. From [HM88, Lemma 12.4], we obtain that $\mathbf{A}$ is right nilpotent, and since the commutator operation in a congruence modular variety is commutative, $\mathbf{A}$ is therefore nilpotent. Now [FM87, Theorem 6.2] yields that $\mathbf{A}$ has a Mal'cev term. Let $\mathbf{A}^{*}$ be the expansion of $\mathbf{A}$ with all its constants. Then $\mathbf{A}^{*}$ is nilpotent and generates a congruence permutable variety. The variety $\mathcal{V}\left(\mathbf{A}^{*}\right)$ is nilpotent by [FM87, Theorem 14.2], and hence congruence uniform by [FM87, Corollary 7.5]. Since for all $n \in \mathbb{N}, \mathbf{F}_{\mathbf{A}^{*}}(n) \leq \mathbf{F}_{\mathbf{A}}(n+|A|) \leq 2^{p(n+|A|)}$, we obtain from the proof of [BB87, Theorem 1] that all commutator terms (in the sense of [Kea99, p. 179]) of $\mathbf{A}^{*}$ are of rank at most $k$. Hence all commutator polynomials 
(in the sense of [AM10, Definition 7.2]) of $\mathbf{A}$ are of rank at most $k$, and then [AM10, Lemma 7.5] yields that $\mathbf{A}$ is $k$-supernilpotent.

For the "only if"-part, we assume that $\mathbf{A}$ is $k$-supernilpotent. Then from the proof of [Wir17, Theorem 4.8], it follows that $\mathbf{A}$ has a Mal'cev term, and thus by Lemma 7.5 of [AM10] each commutator term of $\mathbf{A}$ is of rank at most $k$. Now from the proof of Theorem 1 in [BB87], we obtain a polynomial $p$ of degree at most $k$ such that for all $n \in \mathbb{N}, \mathbf{F}_{\mathbf{A}}(n)$ has exactly $2^{p(n)}$ elements.

Section 4 of [Aic14] contains a self-contained version of Proposition 4.7 for the case that $\mathbf{A}$ is an expanded group.

Corollary 4.8. Let $\mathbf{A}$ be a finite algebra of finite type in a congruence modular variety. Then the following are equivalent.

(1) A is supernilpotent;

(2) there exists constants $c_{1}, c_{2}>0$ such that $\mathrm{h}_{\mathbf{A}}(n) \leq c_{1} \log n+c_{2}$ for all $n>0$;

(3) there exists a polynomial $p_{1}$ such that $\mathrm{l}_{\mathbf{A}}(n) \leq p_{1}(n)$ for all $n>0$;

(4) there exists a polynomial $p_{2}$ such that $\operatorname{Spec}_{\mathbf{A}}(n) \leq 2^{p_{2}(n)}$ for all $n>0$.

Proof. (1) $\Rightarrow(2)$ : as noted in the proof of Proposition 4.7, a finite supernilpotent algebra has a Mal'cev term, therefore Theorem 4.5 applies in this case.

The implications $(2) \Rightarrow(3)$ and $(3) \Rightarrow(4)$ are given by Lemma 2.1 , and $(4) \Rightarrow(1)$ is implied by Proposition 4.7.

Acknowledgements. The authors would like to thank Ágnes Szendrei for supplying the argument leading to Lemma $2.1(3)$.

\section{REFERENCES}

[AE06] Erhard Aichinger and Jürgen Ecker. Every $(k+1)$-affine complete nilpotent group of class $k$ is affine complete. Internat. J. Algebra Comput., 16(2):259-274, 2006.

[Aic14] Erhard Aichinger. On the direct decomposition of nilpotent expanded groups. Comm. Algebra, 42(6):2651-2662, 2014.

[AM10] Erhard Aichinger and Nebojša Mudrinski. Some applications of higher commutators in Mal'cev algebras. Algebra Universalis, 63(4):367-403, 2010.

[BB87] Joel Berman and W. J. Blok. Free spectra of nilpotent varieties. Algebra Universalis, 24(3):279-282, 1987.

[BM14] Wolfram Bentz and Peter Mayr. Supernilpotence prevents dualizability. J. Aust. Math. Soc., 96(1):1-24, 2014.

[BS81] Stanley Burris and Hanamantagouda P. Sankappanavar. A course in universal algebra, volume 78 of Graduate Texts in Mathematics. Springer-Verlag, New York, 1981.

[Bul01] Andrei Bulatov. On the number of finite Mal'tsev algebras. In Contributions to general algebra, 13 (Velké Karlovice, 1999/Dresden, 2000), pages 41-54. Heyn, Klagenfurt, 2001.

[FM87] Ralph Freese and Ralph McKenzie. Commutator Theory for Congruence Modular varieties, volume 125 of London Math. Soc. Lecture Note Ser. Cambridge University Press, 1987.

[HM88] David Hobby and Ralph McKenzie. The structure of finite algebras, volume 76 of Contemporary mathematics. American Mathematical Society, 1988.

[HN15] Gábor Horváth and C. L. Nehaniv. Length of polynomials over finite groups. J. Comput. System Sci., 81(8):1614-1622, 2015.

[HS12] Gábor Horváth and Csaba Szabó. Equivalence and equation solvability problems for the alternating group $\mathbf{a}_{4}$. Journal of Pure and Applied Algebra, 216(10):2170-2176, 2012.

[Kar72] Richard M. Karp. Reducibility among combinatorial problems. In Complexity of Computer Computations (Proc. Sympos., IBM Thomas J. Watson Res. Center, Yorktown Heights, N.Y., pages 85-103. Plenum, New York, 1972.

[Kea99] Keith A. Kearnes. Congruence modular varieties with small free spectra. Algebra Universalis, 42(3):165-181, 1999.

[KP01] Kalle Kaarli and Alden F. Pixley. Polynomial completeness in algebraic systems. Chapman \& Hall / CRC, Boca Raton, Florida, 2001.

[Moo16] Andrew Moorhead. Higher commutator theory for congruence modular varieties. ArXiv e-prints, 2016. https://arxiv.org/abs/1610.07087. 
[Opr16] Jakub Opršal. A relational description of higher commutators in Mal'cev varieties. Algebra universalis, 76(3):367-383, 2016.

[Wir17] Alexander Wires. On supernilpotent algebras. ArXiv e-prints, 2017. https://arxiv.org/abs/1701.08949.

Institut für Algebra, Johannes Kepler Universität Linz, 4040 Linz, Austria

E-mail address: erhard@algebra.uni-linz.ac.at

Department of Mathematics and Informatics, Faculty of Sciences, University of Novi Sad, Trg Dositeja Obradovića 4, 21000 Novi Sad, Serbia

E-mail address: nmudrinski@dmi.uns.ac.rs

Institut für Algebra, Technische Universität Dresden, 01062 Dresden, Germany

E-mail address: jakub.oprsal@tu-dresden.de 